\title{
MOCVD growth of gallium and indium microparticles for SERS applications
}

\author{
Ewa Dumiszewska ${ }^{1, \star}$ (D) Piotr Caban ${ }^{1}$, Iwona Jóźwik ${ }^{1}$, Paweł Ciepielewski ${ }^{1}$, and \\ Jacek M. Baranowski ${ }^{1}$ \\ ${ }^{1}$ Łukasiewicz - Institute of Microelectronics and Photonics, 02-668, al. Lotników 32/46, Warszawa, Poland
}

Received: 5 November 2020

Accepted: 16 February 2021

Published online:

9 March 2021

(C) The Author(s) 2021

\begin{abstract}
The MOCVD growth of Ga and In microparticles was performed on graphene/ $\mathrm{SiC}$ substrates. The test of effectiveness of the microparticles grown for SERS was based on the observation of $\mathrm{H}-\mathrm{Si}$ vibrations on hydrogenated graphene grown on $\mathrm{SiC}$. It was shown by scanning electron microscopy that the Ga or In microparticles grown were in the form of hemispheres with a flat side attached to the substrate. Raman measurements have shown that the effective H-Si SERS signal arises at the edges of the hemisphere microparticles. In addition, it was found that Ga or In microparticles are covered by GaAs or InAs shells, respectively. The presence of GaAs and InAs coverage of metallic microparticles arises from the As contamination of the MOCVD system used for III-V compound growth. However, these coverages do not significantly affect the surface plasmons resonance in the metallic microparticles.
\end{abstract}

\section{Introduction}

Raman probes or sensors that originated from surface-enhanced Raman scattering (SERS) have attracted broad interest in the scientific community due to their unique optical properties and wide applications. Metal nanoparticles have unique optical properties arising from their interaction with an incoming electromagnetic field. They are capable of concentrating and amplifying the electric field in the vicinity of their substrate surfaces. The electron oscillations (plasmons) resonate with light at a certain frequency that is commonly known as the localized surface plasmon resonance (LSPR).
There is an extensive amount of literature devoted to Surface Enhance Raman Scattering (SERS). Some examples of early reviews and original publications are given in Refs. [1-5]. This frequency of oscillations strongly depends on the metal type, the nanoparticle size and their shape [6]. The most commonly studied metal nanoparticles such as silver $(\mathrm{Ag})$ and gold $(\mathrm{Au})$ have their surface plasmon resonance restricted to the visible range [7].

In addition to SERS in $\mathrm{Au}$ and $\mathrm{Ag}$ nanoparticles there has been an effort to search for alternative metals [8-12], in particular Al [13-16], Cu [17], Ni [18] or In [19-21]. In addition to these metals gallium (Ga) has emerged as an interesting plasmonic candidate [22-26]. Gallium nanoparticles can be interesting

Address correspondence to E-mail: ewa.dumiszewska@itme.edu.pl 
since their localized surface plasmon resonances can be tuned from UV to infrared due to the lack of any strong inter-band transitions in this range.

Ga nanoparticles can be grown by Joule-effect thermal evaporation [23, 27] or by the MOCVD method [22]. Both growth methods produce hemispherical nanoparticles in the form of a liquid Ga core and an outer part composed of gallium oxide which maintains the stability of the structure [28]. Ga nanoparticles remain quite stable in the atmosphere due to the existence of a thin native gallium trioxide $\left(\mathrm{Ga}_{2} \mathrm{O}_{3}\right)$ shell which does not significantly affect the LSPRs [28]. Another intriguing characteristic of Ga nanoparticles is the so-called supercooling phenomenon. It means that liquid Ga nanoparticles are transformed into solid ones at a temperature much lower than the melting point of bulk Ga [28]. It has also been discovered that the coupling of Gananoparticles deposited on a graphene/SiC substrate makes it possible to tune SERS from the UV into the near-IR range [25].

Epitaxially grown graphene on $\mathrm{SiC}(0001)$ hydrogenated substrates were used. Epitaxial growth of graphene on $\mathrm{SiC}(0001)$ is connected with the formation of a buffer layer underneath the graphene, which is covalently bound to the underlying $\mathrm{SiC}(0001)$ surface [29]. The electronically inactive buffer layer on $\mathrm{SiC}(0001)$ may be converted into quasi-free-standing monolayer graphene after hydrogen intercalation leaving the $\mathrm{SiC}(0001)$ surface saturated with $\mathrm{H}-\mathrm{Si}$ bonds [29]. The hydrogenated graphene/SiC substrates were used for the epitaxial growth of $\mathrm{Ga}$ and In nanoparticles to disclose the $\mathrm{H}-\mathrm{Si}$ bonds using SERS.

Chemical vapour deposition (CVD) is a technique that consists in depositing a solid material from a gaseous phase on a substrate when a chemical reaction occurs between the gases and the heated substrates. Precursor gases are transported into the reaction chamber at low temperatures. When they come into contact with a heated substrate, they react or decompose forming a solid phase which is deposited onto the substrate [30]. The substrate temperature, the gas flows and the pressure in the reactor are critical parameters and can determine what reactions will take place. MOCVD is the technique mentioned above that uses metalorganic sources as precursors.

\section{Experiment}

Gallium and indium microparticles for SERS were grown on an AIX 200/4 system by metalorganic chemical vapour deposition (MOCVD). $10 \times 10 \mathrm{~mm}$ CVD graphene layers on $4 \mathrm{H}$ and $6 \mathrm{H} \mathrm{SiC}$ were used as substrates. Trimethyl gallium and trimethyl indium were used as gallium and indium precursors, respectively. Nitrogen and hydrogen were used as the carrier gases. The influence of temperature in the reactor and the flow of precursors on the dimension and density of both indium and gallium microparticles was studied. The temperature was varied from 550 to $750{ }^{\circ} \mathrm{C}$. The flow of indium and gallium was kept in the range from 50 to $500 \mathrm{ml} / \mathrm{min}$. and 1 to $10 \mathrm{ml} / \mathrm{min}$. respectively. Indium and gallium microparticles with various dimensions and densities were formed.

The Raman measurements were performed in a backscattering geometry using a Via Renishaw confocal microscope powered by a $532 \mathrm{~nm}(2.33 \mathrm{eV})$ continuous-wave Nd:YAG laser and an $\times 100$ objective. The dimension and density of the microoparticles formed on graphene on $\mathrm{SiC}$ substrates were studied by means of a scanning electron microscope (SEM) technique using the secondary-electron detector of a Hitachi SU8230 high resolution SEM.

\section{Results and discussion}

The Ga and In microparticles grown by MOCVD were investigated with Scanning Electron Microscopy. Figure 1a and b show the surface topography SEM images of graphene on SiC substrates covered with indium and gallium microparticles. The particles are distributed randomly but evenly all over the surface of the substrate. The set of data obtained shows that the distribution and density of microparticles is flow-of-precursors dependent and their density becomes smaller for a lower flow of precursors. The radius of In and Ga microparticles grown on graphene/SiC substrates ranges from $500 \mathrm{~nm}$ to $3 \mu \mathrm{m}$. It was possible to deposit microparticles over the whole range of temperatures from $550^{\circ}$ to $750^{\circ}$ degrees.

Figure 2 shows the SEM images of gallium and indium microparticles taken on graphene/ $\mathrm{SiC}$ substrates at an angle of $54^{\circ}$. The radius of the microparticles is 5 to 10 times longer than that of 
Fig. 1 a SEM image of graphene layer on $\mathrm{SiC}$ substrate covered with gallium

(a) and indium

(b) microparticles

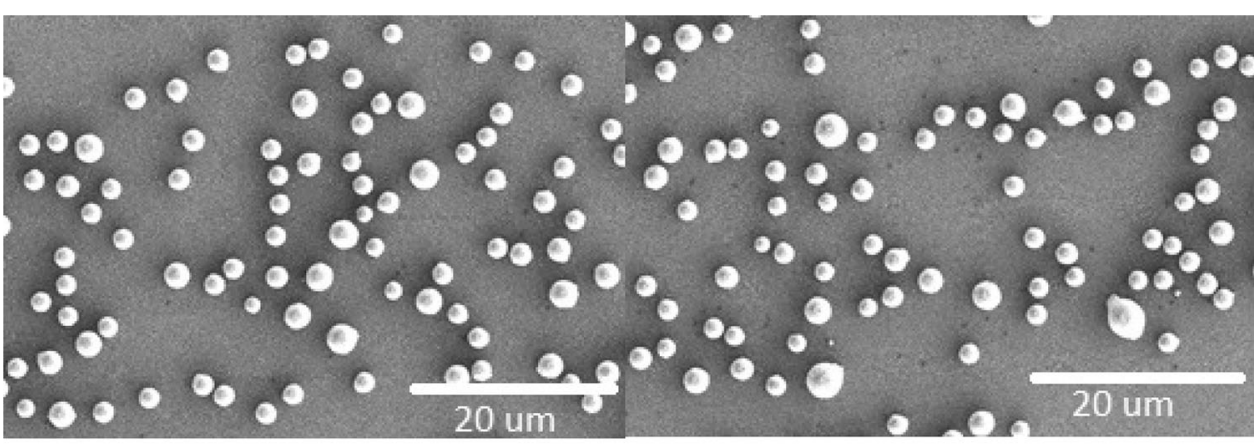

(a) (b) nanoparticles for SERS applications manufactured by other techniques. It is seen that Ga and In microparticles are in the form of hemispheres with a flat side attached to the surface of the graphene layer. One may expect that the roughness of the edges of the hemispheres is responsible for the SERS effect observed.

It is known that in the case of graphene on an $\mathrm{SiC}$ substrate, hydrogen penetrates the buffer layer and the substrate surface and breaks the $s p^{3}$ bonds between the carbon buffer and the silicon substrate atoms. The presence of hydrogen covalently bonded to the Si substrate atoms was discovered by ATR experiments, in which case $\mathrm{Si}-\mathrm{H}$ vibrations at $2128 \mathrm{~cm}^{-1}$ appear [29]. This line becomes much stronger when gold nanoparticles are evaporated and create conditions for SERS observation [31]. The growth of graphene on $4 \mathrm{H}$ and $6 \mathrm{H} \mathrm{SiC}(0001)$ substrates was performed along lines previously developed by us [32]

The SEM image shown in Fig. 2 indicates that the surface of the In hemispheres is covered by some kind of structures. It is possible to identify the chemical composition of the layer covering the surface of metallic Ga and In microparticles by Raman measurements. The Raman results shown in Fig. 3 show characteristic lines due to TO $\left(268 \mathrm{~cm}^{-1}\right)$ and LO $\left(291 \mathrm{~cm}^{-1}\right)$ phonons of GaAs [33]. The formation of a thin layer of GaAs on the surface of $\mathrm{Ga}$ microparticles is due to the residual amount of As in our MOCVD system. Indeed in this MOCVD system about a month ago we performed a process of growing GaAs. This shows that the As memory in the MOCVD system has a very long lifetime. Raman measurements performed on Ga microparticles grown on sapphire gave the same characteristic phonon lines due to GaAs. It was claimed that $\mathrm{Ga}$ microparticles consist of a liquid Ga core and the outer part is composed of gallium oxide which maintains the structure stability [28]. In our case it was found that instead of a native gallium trioxide $\left(\mathrm{Ga}_{2} \mathrm{O}_{3}\right)$ shell, the layer of $\mathrm{GaAs}$ is on the surface of the Ga microparticles. The presence of a thin layer of GaAs most likely protects the Ga microparticles against any future oxidation. The contamination of the MOCVD system used for the growth of III-V compounds may leave some amount of As on the walls of the MOCVD system. During the cooling of

Fig. 2 SEM images of gallium (left image) and indium (right image) microparticles on graphene/ $\mathrm{SiC}$ substrates taken at a $54^{\circ}$ angle

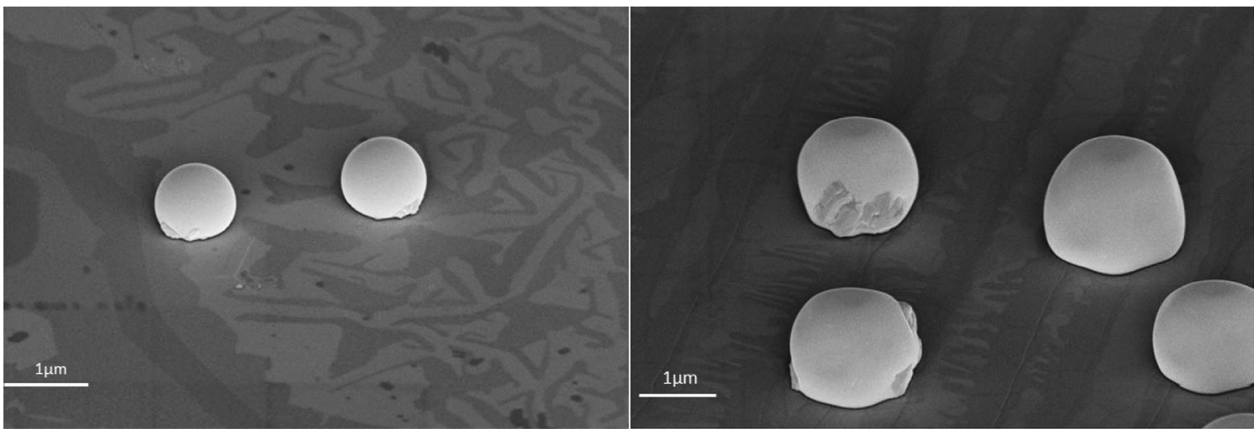




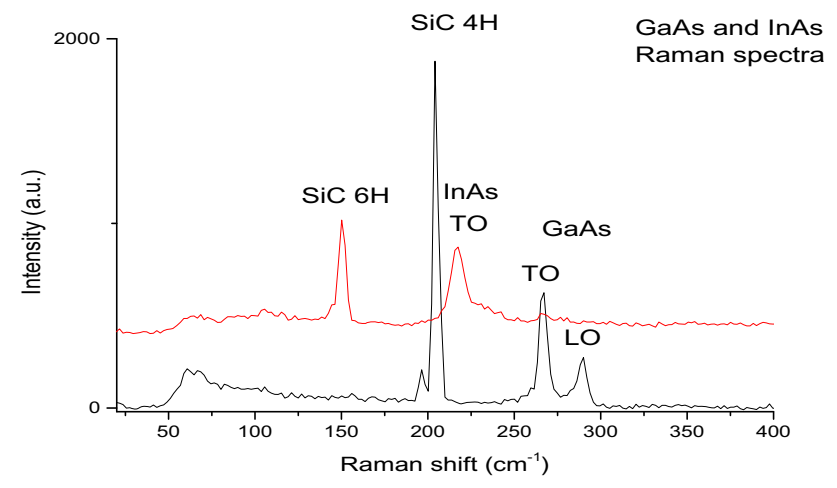

Fig. 3 Raman spectrum of GaAs observed on gallium microparticles grown on graphene/4 HSiC and spectrum of InAs observed on indium microparticles grown on graphene/6H-SiC substrate

the Ga microparticles grown, the As from the MOCVD wall system may be transferred into the microparticles forming the GaAs shell.

Similar Raman measurements have been done on In microparticles. In this case a line which is characteristic for InAs due to TO $\left(220 \mathrm{~cm}^{-1}\right)$ phonon and LO phonon in the form of a shoulder has been observed [34]. The InAs TO line has been observed in In microparticles grown on graphene/SiC and sapphire substrates as well. The formation of InAs outside In microparticles has not been observed.

In order to study the influence of the microparticles deposited on the $\mathrm{Si}-\mathrm{H}$ line, Raman spectroscopy measurements were performed on hydrogenated graphene layers with $\mathrm{Ga}$ and In microparticles. The comparison of the spectra is presented in Fig. 4.

The investigations conducted show that $\mathrm{H}-\mathrm{Si}$ vibrations can be observed when either Ga or In microparticles are present on the graphene/SiC surface, creating suitable conditions for the SERS effect. The line at $2128 \mathrm{~cm}^{-1}(\mathrm{H}-\mathrm{Si})$ appears in the spectra in the vicinity of either Ga or In microparticles on the graphene surface. The magnification of the $\mathrm{H}-\mathrm{Si}$ line by plasmonic effect indicates that $\mathrm{Ga}$ and $\mathrm{In}$ microparticles are indeed metallic and the presence of GaAs and InAs layers on them does not significantly affect the LSPRs. It should be noted that only $\mathrm{H}-\mathrm{Si}$ vibrations, which are perpendicular to the graphene surface, are SERS active. On the contrary, the graphene $\mathrm{G}$ and 2D modes, which are extended and lie in the graphene plane are not SERS magnified.

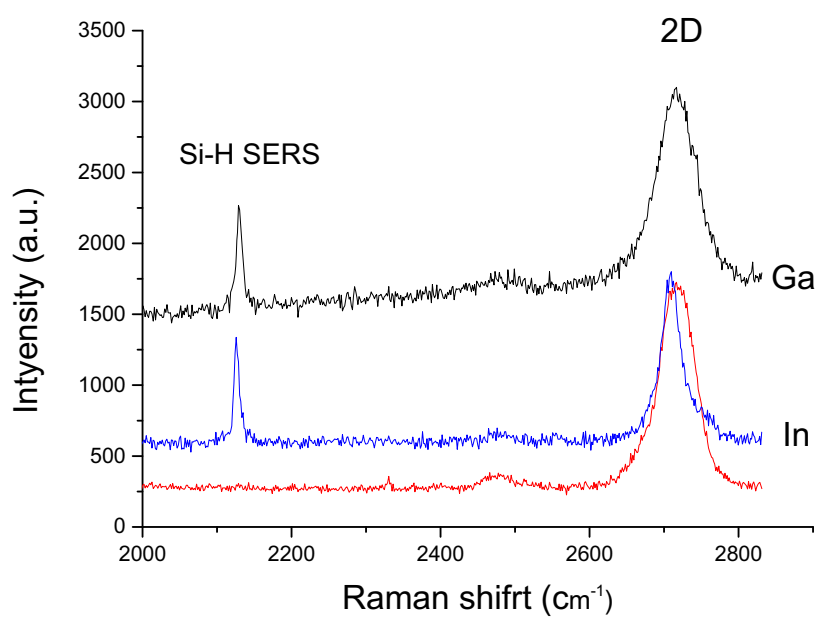

Fig. 4 Raman spectra taken in vicinity of Ga and In microparticle on graphene/ $\mathrm{SiC}$ substrates and between microparticles (red line). 2D peak of graphene is indicated (Color figure online)

The intensity of the $2128 \mathrm{~cm}^{-1}$ peak (H-Si vibrations) dependence on the beam position in respect of the microparticle (optical image in Fig. 5a) is presented in Fig. 5b It clearly confirms that the SERS effect is observable only in the close proximity of Ga or In microparticles.

This conclusion is supported by Raman measurements in polarized light as shown in Fig. 6.

The SERS measurements on the isolated microparticle in polarized light show that the central part of the microparticle is not active. The polarization results presented in Fig. 6 show that H-Si SERS occur on the edges of the microparticle and in areas where the polarization of the laser beam is perpendicular to the edge of the hemisphere. This suggests that plasmons in the hemisphere are generated along the polarization of the light. The asymmetry of the SERS H-Si signal on the edges of the Ga microparticle visible in Fig. 6 suggest that either the roughness on the edges of the microparticle or the density of the $\mathrm{H}-$ $\mathrm{Si}$ on the graphene/SiC is not uniform.

\section{Summary}

The MOCVD growth of Ga and In microparticles on graphene/SiC substrates is presented. The SERS measurements connected with the Ga and In microparticles grown by the MOCVD on graphene/ 
Fig. 5 a The optical microscope image of microparticles on graphene/ $\mathrm{SiC}, \mathbf{b}$ map of intensity of Raman H-Si $2128 \mathrm{~cm}^{-1}$ registered in the same area as shown in the image a
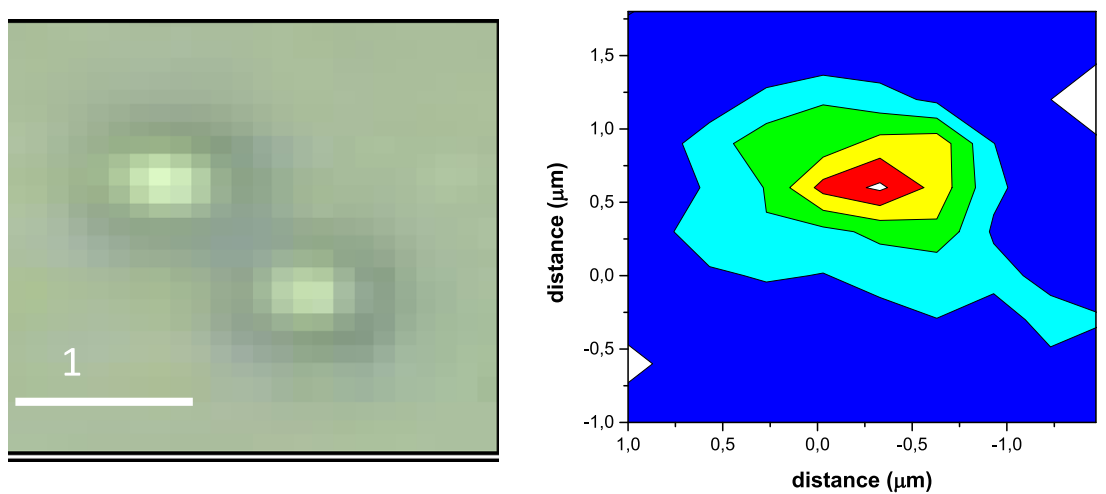

H-Si mode intensity

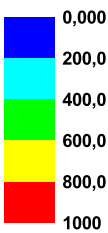

(a.u.)
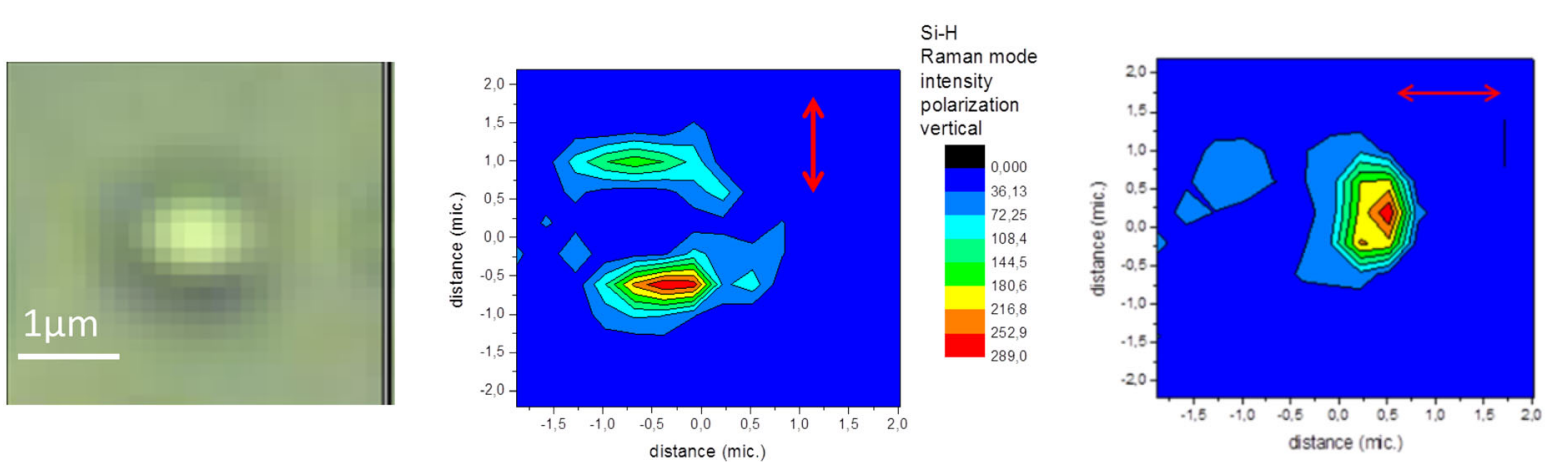

Si-H

Raman mode

intensity

polarization

horizon

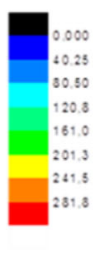

Fig. 6 Optical image of isolated Ga microparticle on graphene/SiC and Raman measurements of intensity of $\mathrm{H}-\mathrm{Si}$ line taken in polarized light. Polarization of light is indicated by arrow

$\mathrm{SiC}$ substrates reveal $\mathrm{H}-\mathrm{Si}$ vibrations. The microparticles grown are hemispheres with their flat side attached to the graphene/SiC substrate. The most important conclusion of these investigations is connected with the demonstration that relatively large microparticles of sizes of about $0.5-3 \mu \mathrm{m}$ can be used for SERS measurements. The coverage of Ga or In microparticles by GaAs or InAs shells does not significantly affect the surface plasmon resonance. The presence of As most likely originates from the memory effect of the MOCVD system. It should be noticed that the growth of Ga or In microparticles on sapphire can be used as a test for the purity of the MOCVD system.

The investigation presented shows that indium and gallium microparticles deposited on various substrates by means of MOCVD may be good candidates for SERS substrates.
Open Access This article is licensed under a Creative Commons Attribution 4.0 International License, which permits use, sharing, adaptation, distribution and reproduction in any medium or format, as long as you give appropriate credit to the original author(s) and the source, provide a link to the Creative Commons licence, and indicate if changes were made. The images or other third party material in this article are included in the article's Creative Commons licence, unless indicated otherwise in a credit line to the material. If material is not included in the article's Creative Commons licence and your intended use is not permitted by statutory regulation or exceeds the permitted use, you will need to obtain permission directly from the copyright holder. To view a copy of this licence, visit http://creativecommons.org/licen ses $/$ by $/ 4.0 /$.

\section{References}

1. M. Moskovits, Surface-enhanced spectroscopy. Rev. Mod. Phys. 57, 783-826 (1985) 
2. M.I. Stockman, V.M. Shalaev, M. Moskovits, R. Botet, T.F. George, Enhanced Raman scattering by fractal clusters: scale invariant theory. Phys. Rev. B 46, 2821-2830 (1992)

3. L. Gunnarsson, S. Petronis, B. Kasemo, H. Xu, J. Bjerneld, M. Kall, Optimizing nanofabricated substrates for surface enhanced Raman scattering. Nanostruct. Mater. 12, 783-788 (1999)

4. H.X. Xu, E.J. Bjerneld, M. Kall, L. Borjesson, Spectroscopy of single hemoglobin molecules by surface enhanced Raman scattering. Phys. Rev. Lett. 83, 4357-4360 (1999)

5. H. Xu, J. Aizpurua, M. Kall, P. Apell, Electromagnetic contributions to single-molecule sensitivity in surface-enhanced Raman scattering. Phys. Rev. E 62, 4318-4324 (2000)

6. M.A. García, Surface plasmons in metallic nanoparticles: fundamentals and applications. J. Appl. Phys. 45, 389501 (2012). https://doi.org/10.1088/0022-3727/45/38/389501

7. F. Hubenthal, T. Ziegler, C. Hendrich, M. Alschinger, F. Träger, Tuning the surface plasmon resonance by preparation of gold-core/silver-shell and alloy nanoparticles. Eur. Phys. J. D 34, 165-168 (2005). https://doi.org/10.1140/epjd/e200500138-1

8. G.V. Naik, V.M. Shalaev, A. Boltasseva, Alternative plasmonic materials: beyond gold and silver. Adv. Mat. 25, 3264-3294 (2013). https://doi.org/10.1002/adma.201205076

9. P.R. West et al., Searching for better plasmonic materials. Laser Photon. Rev. 4, 795-808 (2010). https://doi.org/10.10 02/lpor.200900055

10. W.T. Hsieh et al., Comparative analysis of metals and alternative infrared plasmonic materials. ACS Photon. 5, 2541-2548 (2018). https://doi.org/10.1021/acsphotonics.7b 01166

11. J.M. Sanz et al., UV plasmonic behavior of various metal nanoparticles in the near- and far-field regimes: geometry and substrate effects. J. Phys. Chem. C 117, 19606-19615 (2013). https://doi.org/10.1021/jp405773p

12. J.M. McMahon, G.C. Schatz, S.K. Gray, Plasmonics in the ultraviolet with the poor metals $\mathrm{Al}, \mathrm{Ga}, \mathrm{In}, \mathrm{Sn}, \mathrm{Tl}, \mathrm{Pb}$, and $\mathrm{Bi}$. Phys. Chem. Chem. Phys. 15, 5415-5423 (2013). https://doi. org/10.1039/C3CP43856B

13. K. Diest, V. Liberman, D.M. Lennon, P.B. Welander, M. Rothschild, Aluminum plasmonics: optimization of plasmonic properties using liquid-prism-coupled ellipsometry. Opt. Express 21, 28638-28650 (2013). https://doi.org/10.13 64/OE.21.028638

14. G.H. Chan, J. Zhao, G.C. Schatz, R.P. Van Duyne, Localized surface plasmon resonance spectroscopy of triangular aluminium nanoparticles. J. Phys. Chem. C 112, 13958-13963 (2008). https://doi.org/10.1021/jp804088z
15. M.W. Knight, N.S. King, L. Liu, H.O. Everitt, P. Nordlander, N.J. Halas, Aluminum for plasmonics. ACS Nano 8, 834-840 (2014). https://doi.org/10.1021/nn405495q

16. M.W. Knight, L.F. Liu, Y.M. Wang, L. Brown, S. Mukherjee, N.S. King, H.O. Everitt, P. Nordlander, N.J. Halas, Aluminum plasmonic nanoantennas. Nano Lett. 12, 6000-6004 (2012). h ttps://doi.org/10.1021/n1303517v

17. H. Wang, F. Tam, N.K. Grady, N.J. Halas, Cu Nanoshells: effects of interband transitions on the nanoparticle plasmon resonance. J. Phys. Chem. B 109, 18218-18222 (2005). h ttps://doi.org/10.1021/jp053863t

18. S. Schuermans, T. Maurer, J. Martin, J.-B. Moussy, J. Plain, Plasmon/interband transitions coupling in the UV from large scale nanostructured Ni films. Opt. Mater. Express 7, 1787-1793 (2017). https://doi.org/10.1364/OME.7.001787

19. S. Cingarapu, Z. Yang, C.M. Sorensen, K.J. Klabunde, Synthesis of indium nanoparticles: digestive ripening under mild conditions. Inorg. Chem. 50, 5000-5005 (2011). https://doi. org/10.1021/ic200232b

20. T.H. Lim, B. Ingham, K.H. Kamarudin, P.G. Etchegoin, R.D. Tilley, Solution synthesis of monodisperse indium nanoparticles and highly faceted indium polyhedra. Cryst. Growth Des. 10， 3854-3858 (2010). https://doi.org/10.1021/cg $901562 d$

21. K. Krane, H. Raether, Measurement of surface plasmon dispersion in aluminum and indium. Phys. Rev. Lett. 37, 1355-1357 (1976). https://doi.org/10.1103/PhysRevLett.37. 1429

22. L. Imhoff, O. Heintz, V. Gauthier, L. de Marco, S. Bourgeois, Chemical characterization of gallium droplets grown by LPMOCVD. Appl. Surf. Sci. 253, 2820 (2006). https://doi.org/ 10.1016/japsusc206.05.062

23. S. Catalan-Gomez, C. Bran, M. Vazquez, J.L. Pau, A. Redondo-Cubero, Plasmonic coupling in closed-packed ordered gallium nanoparticles. Sci. Rep. 10, 4187 (2020)

24. P.C. Wu et al., Demonstration of surface-enhanced Raman scattering by tunable, plasmonic gallium nanoparticles. J. Am. Chem. Soc. 131, 12032-12033 (2009). https://doi.org/ 10.1021/ja903321z

25. C. Yi et al., Evidence of plasmonic coupling in gallium nanoparticles/graphene/SiC. Small 8, 2721-2730 (2012). h ttps://doi.org/10.1002/smll.201200694

26. J.L. Pau, A. García-Marín, M.J. Hernández, E. Lorenzo, J. Piqueras, Optical biosensing platforms based on Ga-graphene plasmonic structures on $\mathrm{Cu}$, quartz and $\mathrm{SiO} 2 / \mathrm{Si}$ substrates. Physica Status Solidi (b) 253, 664-670 (2016). https://doi. org/10.1002/pssb.201552493

27. S. Catalán-Gómez et al., Size-selective breaking of the coreshell structure of gallium nanoparticles. Nanotechnology 29, 355707 (2018) 
28. Y. Gutierrez et al., How an oxide shell affects the ultraviolet plasmonic behavior of $\mathrm{Ga}, \mathrm{Mg}$, and $\mathrm{Al}$ nanostructures. Opt. Express. 24, 20621-20631 (2016). https://doi.org/10.1364/O E.24.020621

29. C. Riedl, C. Coletti, T. Iwasaki, A.A. Zakharov, U. Starke, Quasi-free-standing epitaxial graphene on $\mathrm{SiC}$ obtained by hydrogen intercalation. Phys. Rev. Lett. 103, 246804 (2009). https://doi.org/10.1103/PhysRevLett.103.246804

30. M. Ohring, Materials Science of Thin Films (Academic Press, London, 2002).

31. N. Ferralis, C. Carraro, Evolution of interfacial intercalation chemistry on epitaxial graphene/SiC by surface enhanced Raman spectroscopy. Appl. Surf. Sci. 320, 441-447 (2014). h ttps://doi.org/10.1016/j.apsusc.2014.09.096
32. W. Strupinski, K. Grodecki, A. Wysmolek, R. Stepniewski, T. Szkopek, P.E. Gaskell, A. Grüneis, D. Haberer, R. Bozek, J. Krupka, J.M. Baranowski, Graphene epitaxy by chemical vapor deposition on SiC. Nano Lett. 11(4), 1786-1791 (2011). https://doi.org/10.1021/nl200390e

33. A. Mooradian, G.B. Wright, Observation of the interaction of plasmons with longitudinal optical phonons in GaAs. Phys. Rev. Lett. 16, 999 (1969)

34. R.C.C. Leite, J.F. Scott, Resonant surface Raman scattering in direct-gap semiconductors. Phys. Rev. Lett. 22, 130 (1969)

Publisher's Note Springer Nature remains neutral with regard to jurisdictional claims in published maps and institutional affiliations. 